\title{
International collaboration in disposal research: comparative modeling of coupled processes in the DECOVALEX project
}

\author{
Jens T. Birkholzer ${ }^{1}$ and Alex Bond ${ }^{2}$ \\ ${ }^{1}$ Energy Geosciences Division, Lawrence Berkeley National Laboratory, Berkeley, USA \\ ${ }^{2}$ Quintessa Ltd., Warrington, UK \\ Correspondence: Jens T. Birkholzer (jtbirkholzer@lbl.gov)
}

Published: 10 November 2021

\begin{abstract}
This presentation gives an overview of an international research collaboration for advancing the understanding and modeling of coupled thermo-hydro-mechanical-chemical (THMC) processes in geological systems. The creation of the international DECOVALEX project, now running for more than 25 years, was motivated by the recognition that prediction of these coupled effects is an essential part of the performance and safety assessment of geologic disposal systems for radioactive waste and spent nuclear fuel. DECOVALEX emphasizes joint analysis and comparative modeling of state-of-the-art field and laboratory experiments, across a range of host rock options and repository designs. Participating research teams are from radioactive waste management organizations, national research institutes, regulatory agencies, universities, and consulting groups, providing a wide range of perspectives and solutions to these complex problems. The presentation provides examples of the research contributions made collectively in past DECOVALEX tasks and also touches on the unique modeling challenges tackled in the ongoing project phase, referred to as DECOVALEX-2023. The current phase comprises 17 partner organizations, about 50 modeling teams, and 7 modeling tasks, which cover a broad portfolio from fundamental studies on gas migration to full-scale in situ heater experiments in different host rocks to performance assessment studies. Together, these examples illustrate that the insight and scientific knowledge gained within the DECOVALEX project would not have been possible if one group had studied these complex THMC modeling challenges alone rather than within a truly collaborative setting.
\end{abstract}

Kurzfassung. Dieser Vortrag gibt einen Überblick über eine internationale Forschungskooperation zur Förderung des Verständnisses und der Modellierung von gekoppelten thermo-hydro-mechanisch-chemischen (THMC) Prozessen in geologischen Systemen. Die Motivation zur Gründung des internationalen DECOVALEXProjekts, das nun seit mehr als 25 Jahren läuft, entstand aus der Erkenntnis heraus, dass die Vorhersage dieser gekoppelten Effekte einen wesentlichen Bestandteil der Sicherheitsbewertung von geologischen Endlagersystemen für radioaktive Abfälle und abgebrannte Brennelemente darstellt. DECOVALEX legt den Schwerpunkt auf die gemeinsame Analyse und vergleichende Modellierung modernster Feld- und Laborexperimente für eine Reihe von Wirtsgesteinsoptionen und Endlagerkonzepten. Die teilnehmenden Forschungsteams kommen aus Organisationen für die Entsorgung radioaktiver Abfälle, nationalen Forschungsinstituten, Aufsichtsbehörden, Universitäten und Beratungsunternehmen und bieten eine breite Palette von Perspektiven und Lösungen für diese komplexen Probleme. Die Präsentation enthält Beispiele für die Forschungsbeiträge, die im Rahmen früherer DECOVALEX-Aufgaben gemeinsam geleistet wurden, und geht auch auf die einzigartigen Modellierungsherausforderungen ein, die in der laufenden Projektphase, welche als DECOVALEX-2023 bezeichnet wird, angegangen werden. Die aktuelle Phase umfasst 17 Partnerorganisationen, etwa 50 Modellierungsteams und 7 Modellierungsaufgaben, die ein breites Spektrum abdecken, von grundlegenden Studien zur Gasmigration über In-situHeizexperimente in verschiedenen Wirtsgesteinen bis hin zu Studien zur Sicherheitsbewertung. Diese Beispiele 
zeigen, dass die im Rahmen des DECOVALEX-Projekts gewonnenen Einsichten und wissenschaftlichen Erkenntnisse nicht möglich gewesen wären, wenn die Untersuchung dieser komplexen THMC-Modellierungsaufgaben nur durch eine Gruppe alleine und nicht in einem echten gemeinschaftlichen Rahmen erfolgt wäre.

Financial support. This research has been supported by the U.S.

Department of Energy (grant no. DE-AC02-05CH11231). 